\title{
EL HOMBRE EN LA NATURALEZA: LOS RESULTADOS DEL Proyecto Chicago en Chiapas, 1956-1959
}

\author{
Man in Nature: Results of The Chicago \\ PROJeCt IN ChIAPAS, 1956-1959
}

Óscar Javier Barrera-Aguilera*

Resumen: En este artículo se analizan los resultados a los que llegó el equipo de trabajo del proyecto desarrollado por la Universidad de Chicago sobre las poblaciones tseltales y tsotsiles de Chiapas, concentrándose en su primera fase operativa (1956-1959) y en los documentos que ofrecieron visiones de conjunto. Se revisan las características generales de dicha empresa intelectual, se presenta cómo ha sido entendido el proyecto desde la disciplina antropológica y se ofrece una propuesta alternativa para su comprensión. Para finalizar, se estudian los aportes y contradicciones ofrecidos en el reporte y se muestran al lector elementos para revalidar las actividades emprendidas por el proyecto Chicago en Chiapas.

Palabras clave: cambio cultural, historia de la antropología, ladinización, Proyecto Man-in-Nature, tseltales, tsotsiles.

Abstract: This article analyzes the results of the first operational phase (1956-1959) of a University of Chicago project that focused on the Tseltal and Tsotsil populations of Chiapas, and the documents that offered shared visions. First, we deal with the general characteristics of this intellectual endeavor. Second, we discuss the usual way anthropologists have understood the project and we present an alternative perspective. Third, we explore the contributions and contradictions offered. The last section discusses elements that validate the activities undertaken by the Chicago Project in Chiapas.

Keywords: cultural change; history of anthropology; ladinoization; Man-in-Nature Project; Tseltals; Tsotsils.

\footnotetext{
* Óscar Javier Barrera Aguilera. Doctor en Historia por El Colegio de México, México. Becario del Programa de Becas Posdoctorales en la UNAM, Centro de Investigaciones Multidisciplinarias sobre Chiapas y la Frontera Sur, México, asesorado por el doctor Gabriel Ascencio Franco. Temas de especialización: historia de la ladinización en Chiapas y de la antropología en América Latina. Correo electrónico: cayatano68@yahoo.com. ORCID: orcid.org/0000-0002-7704-9596.

Enviado a dictamen: 13 de marzo de 2018

Aprobación: 17 de octubre de 2018

Revisiones: 1
} 


\section{El objeto de investigación}

E ntre 1956 y 1962, la Universidad de Chicago emprendió uno de los proyectos más tempranos, sistemáticos e incluyentes del que tengamos noticia sobre los pueblos ubicados en la zona central de Chiapas (ver Mapa 1). "El hombre en la naturaleza" (Man in Nature) fue un proyecto que tuvo como objetivo original investigar las relaciones que las comunidades tseltales y tsotsiles tenían con su medioambiente. En 1959, Norman McQuown, uno de sus directores, promocionaba el proyecto como el primer estudio dialectal y geográfico de las variaciones del habla en sociedades indígenas que se había realizado hasta entonces. En realidad fue más que eso. Consistió en un trabajo colectivo multidimensional y multidisciplinario que también abarcó pueblos de otras regiones y del que curiosamente fueron pocos los resultados que se dieron a conocer públicamente (McQuown y PittRivers, 1970). ${ }^{2}$

El proyecto Chicago se desarrolló en dos etapas. A una primera fase pertenecen los informes que el equipo de investigadores agrupó en el reporte presentado en junio de 1959, correspondiente a las actividades adelantadas desde 1956. En el mismo se recogieron trabajos sobre el hábitat, la vegetación, la arqueología, la lingüística y la etnohistoria de la zona, los cuales fueron acompañados por monografías de los pueblos de Amatenango del Valle, Aguacatenango, Chanal, Teopisca, Venustiano Carranza, Villa Las Rosas, Oxchuc y Huixtán. Este primer avance del equipo de investigación a su vez involucró trabajos comparativos y de profundización de algunos temas en particular. ${ }^{3}$ La segunda fase del proyecto (1959-1962) se propuso estudiar el cambio cultural producido en el presente y el proceso etnohistórico de transformación. Los productos más conocidos de esa etapa son los artículos y libros publicados con posterioridad por la antropóloga Esther Hermitte y la edición póstuma de algunos escritos de Marcelo Díaz de Salas (Medina, 1995).

Esta propuesta pretende analizar los productos del proyecto Chicago en su primera fase. El principal objetivo es tratar de ubicar históricamente la empresa académica mencionada y así identificar sus fundamentos y propósitos, el desarrollo del proceso investigativo y los resultados alcanzados.

\section{El contexto}

\section{La visión ortodoxa}

Durante el gobierno de Adolfo López Mateos (19581964) la antropología fue de la mano del indigenismo y del nacionalismo (Medina, 1983). Al mismo tiempo, las universidades norteamericanas dominaron el trabajo de campo en Chiapas y defendieron el indigenismo como un modelo de antropología aplicada (Fábregas, 2007). Éste fue el contexto general de la política y la antropología mexicanas que recibió a los investigadores participantes en proyectos como aquellos que abanderaron las universidad de Chicago y Harvard hacia 1960. No obstante, queda preguntarse de qué manera concreta estas condiciones determinaron los objetivos y las prácticas de estas empresas académicas.

Por otra parte, es un lugar común señalar que hasta los años sesenta del siglo pasado dominó en la antropología mexicana el enfoque culturalista norteamericano, el cual fue fuertemente cuestionado desde la teoría crítica marxista. Con la Revolución cubana se alimentó un movimiento político que denunció el profundo colonialismo dominante entre la mayoría de los intelectuales latinoamericanos. En la antropología mexicana aparecieron los trabajos de Guillermo Bonfil, Pablo González Casanova y Rodolfo Stavenhagen, los cuales cuestionaron el culturalismo y propusieron que la disciplina se ocupara de la población indígena teniendo en cuenta la estructura socioeconómica del país (Medina, 1983). Stavenhagen particularmente cuestionó las monografías culturalistas desarrolladas en Los Altos de Chiapas, que se ocuparon de las relaciones entre indígenas y ladinos, pues — según él- descuidaban aspectos como la distribución de la tierra, así como también las relaciones de trabajo, comercio y propiedad que ligaban a una parte de la población con la otra (Medina, 1983, 2015). 


\section{Una propuesta revisionista}

Consideramos que el mismo desarrollo de la antropología y una revisión de los argumentos y resultados presentados por los participantes en el proyecto Chicago podrían llevar a cuestionar la utilidad y el valor explicativos que etiquetas como "culturalismo", "estructural-funcionalismo" o "estudios de comunidad" ofrecen para comprenderlo y ponderar los resultados que obtuvo. Alfonso Villa Rojas y varios antropólogos nacionales y extranjeros que trabajaron en Los Altos de Chiapas se apoyaron en la antropología social británica y en Robert Redfield, quien exhibió una clara orientación sociológica. A finales de la década de 1960, Ángel Palerm apuntó en esta última dirección, cuando hizo una revaloración de la antropología social, reconociendo la diversidad de tendencias teóricas en la antropología norteamericana (Fábregas, 2007: 26).

En los años cincuenta, las universidades de Harvard y Chicago llevaron a cabo proyectos interdisciplinarios de largo aliento en Chiapas. En el caso del primer proyecto contamos con un trabajo que recoge la memoria personal de su principal mentor. El proyecto Chiapas de Harvard inició en 1957 y estuvo en operación por 35 años. El proyecto se enfocó en los municipios indígenas hablantes de tsotsil ubicados en Los Altos de Chiapas. En 1955, Evon Vogt fue invitado por Alfonso Caso a un congreso destinado a evaluar los programas del Instituto Nacional Indigenista (Vogt, 1994).

Vogt contó con la fortuna de iniciar su proyecto en un momento en el que Alfonso Villa Rojas estaba al comando del programa local de gobierno dirigido a los indígenas. El proyecto presentado por Vogt estaba interesado en un análisis comparativo de los procesos de cambio cultural en comunidades tsotsiles y tseltales. En gran medida, se trataba de estudiar el impacto de las políticas implementadas por el INI a partir de la década de 1950 (Medina, 2015; Vogt, 1994).

El coordinador del proyecto Harvard admitió que, al haberse formado bajo la influencia de profesores como Robert Redfield y Sol Tax, el énfasis estaba puesto en fenómenos sociales y culturales al interior de comunidades. No obstante, Vogt también señaló que debía reconocerse que hubo trabajos referentes a las relaciones entre indígenas y ladinos (como el de Barbara Metzger) o a las conexiones económicas que se tejieron entre los pueblos (como los de Frank Cancian). Además, Vogt apuntó que en el caso del proyecto Chicago hubo trabajos comparativos entre pueblos tsotsiles y tseltales, como los desarrollados por Norman McQuown y Julian Pitt-Rivers, e incluso se produjeron algunos dedicados a estudiar las relaciones de poder al interior de los pueblos, como el de Michael Salovesh para el caso de Venustiano Carranza (Medina, 2015; Vogt, 1994).

Andrés Fábregas Puig recientemente ha propuesto trascender la manera como han sido valorados los trabajos de los investigadores que participaron en los proyectos de origen norteamericano. En los textos de Esther Hermitte — como en los de muchos otros de sus colegas - no aparece la clásica comunidad cerrada sino la interacción de los indígenas con los ladinos y la modernización del país (Fábregas, 2007).

Gran parte de las investigaciones que se emprendieron bajo el proyecto Chicago se propusieron examinar los procesos de cambio social y cultural de los indígenas tseltales y tsotsiles, enfocándose en la "ladinización", es decir, el pasaje de "indio" a "ladino", que implicaba una modificación del estatus social que, entre otras cosas, podía entrañar el abandono de la lengua, el cambio de vestimenta o la renuencia a participar en la jerarquía cívico-religiosa (Guber, 2007). Lejos del molde estructural-funcionalista, que supone pueblos estáticos y cerrados, y con el que usualmente han sido catalogados los resultados de las investigaciones (Medina, 2015), los trabajos de los estudiosos que participaron en el proyecto Chicago encararon las relaciones cambiantes y conflictivas entre indígenas y ladinos.

\section{Generalidades, comparaciones y resultados}

\section{Los hallazgos, las tipologías y el cambio cultural}

En un apartado conjunto, Manning Nash, Muriel Verbitsky, John Hotchkiss y Norman McQuown ofrecieron una visión esquemática de las poblaciones 
que fueron objeto de estudio. El criterio básico para establecer la clasificación fue el grado de retención o asimilación que mostraban los pueblos investigados con respecto a tres momentos de contacto: por una parte, la cultura y organización social de los mayas tempranos; por otra, las características introducidas por los españoles en los primeros tiempos coloniales; $y$, por último, los rasgos prestados de la cultura nacional mexicana contemporánea. ${ }^{4}$

De acuerdo con lo anterior, los investigadores identificaron tres tipos de pueblos: unos que clasificaron como comunidades corporadas - entre ellos Chanal, Amatenango y Aguacatenango-, es decir, con mecanismos que perseguían la homogeneidad económica y social; otros que consideraron en una situación intermedia, como Venustiano Carranza, consistente en una larga convivencia con los ladinos que, si bien había provocado la eliminación de algunas características del corporativismo, no había conducido a la fragmentación y diferenciación de la comunidad indígena; y algunos, como Teopisca, caracterizados como comunidades abiertas en las que dominaban los ladinos y sus indígenas eran migrantes recientes de orígenes muy diversos. ${ }^{5}$

Norman McQuown expuso algunas de las principales novedades y conclusiones a las que llegó el proyecto en su primera fase. Probablemente la mayor originalidad de esta empresa académica consistió en haber ofrecido el primer estudio dialectal y geográfico de la variación del habla en un grupo indígena. Quizás la anterior especificidad se queda corta al momento de describir los alcances finales que tuvo la propuesta, pues su principal fortaleza provino de reunir investigadores provenientes de diferentes disciplinas con la finalidad de analizar múltiples dimensiones de los pueblos tseltales y tsotsiles de Chiapas. ${ }^{6}$

Entre las conclusiones ofrecidas por Norman en el primer informe destaca la correlación entre individuo y tierra. La posesión de cantidades desproporcionadas de tierra por parte de una o varias personas podría generar un desequilibrio de riqueza que, a la postre, contribuiría a debilitar la comunidad corporada. En esta misma línea analítica, y en aras de preservar el corporativismo, las comunidades podían generar mecanismos para restringir la acumulación de poder personal proveniente de la posesión de tierra. En esta dirección apuntarían adaptaciones tales como la especialización de las actividades al interior de la comunidad, la redefinición de la marginalidad social y el incremento de las acusaciones por brujería, o la migración de parte de la fuerza de trabajo hacia otros lugares vecinos y lejanos. ${ }^{7}$

Para la elaboración de uno de los ensayos comparativos John Hotchkiss acudió a los documentos incluidos en este mismo reporte referentes a los pueblos de Chanal, Teopisca y Venustiano Carranza. Quisiera resaltar un par de aspectos que se desprenden de la lectura de este trabajo. Por una parte, en este estudio comparativo el autor reveló el profundo desconocimiento que hasta entonces se tenía de los procesos históricos experimentados por Chanal, Teopisca y Venustiano Carranza. Por otra, en el texto se propuso que el declive comercial del último pueblo mencionado fue resultado de la construcción de la carretera Panamericana, la cual habría cambiado los flujos económicos. ${ }^{8}$

John Hotchkiss dio prioridad a las carreteras como factores de cambio. No obstante, las investigaciones posteriores de Juan María Morales Avendaño y Virginia Molina propondrían que el estancamiento del pueblo entonces conocido como San Bartolomé de Los Llanos fue consecuencia en gran medida de los cambios de poder desde la ciudad de San Cristóbal de Las Casas hacia la de Tuxtla Gutiérrez a finales del siglo XIX (Molina, 1976; Morales, 1986). Nuestras propias pesquisas nos han revelado que el declive del pueblo otrora más grande de la Depresión Central habría sido motivado por una serie de epidemias que provocaron muchas muertes y la consecuente migración hacia las fincas de los Cuxtepeques durante la década de 1850 (Barrera, 2016).

\section{El medio y los hombres}

Philip Wagner y John Hotchkiss compararon diferentes pueblos en un documento. Los autores señalaron un 
aspecto poco atendido: la retirada de los ladinos de algunos pueblos de las tierras altas. Los investigadores resaltaron una diferencia importante con respecto a la tenencia y calidad de la tierra entre las comunidades mezcladas - como Venustiano Carranza y Teopisca-y los pueblos con mayoría indígena - como Amatenango, Aguacatenango y Villa Las Rosas-: mientras en el primer caso las tierras de los indígenas eran de menor calidad y estaban separadas de las tierras de los ladinos, en el segundo la autonomía política y económica estaba asociada al control de las tierras comunales. ${ }^{9}$

Philip Wagner comparó las relaciones sostenidas entre pueblos ubicados en diferentes hábitats. Uno de los puntos fundamentales de la argumentación consistió en describir los lugares de habitación y las actividades productivas asociadas a los mismos. Los pueblos de Teopisca, Villa Las Rosas y Venustiano Carranza fueron identificados porque la mayoría de las actividades dirigidas a ofrecer servicios eran realizadas por población no indígena. Por otra parte, Philip Wagner recalcó la diversidad de formas de habitación practicadas por las poblaciones indígenas: desde aquellas que vivían en pueblos propiamente suyos — como en Amatenango y Aguacatenango-; pasando por quienes residían en parajes ubicados en la periferia de los pueblos ladinos — como en Teopisca-; e incluyendo a las personas que habitaban en barrios al interior de los pueblos - como ocurría en Teopisca, Carranza y Villa Las Rosas-. Desde luego que dichos pueblos y sus habitantes no se hallaban del todo aislados: muchos indígenas de Amatenango, Aguacatenango y varios parajes acudían a Teopisca, Villa Las Rosas y Carranza para intercambiar productos agrícolas y bienes de primera necesidad. ${ }^{10}$

Otro trabajo de tipo comparativo fue el que redactó Lawrence Kaplan. El aspecto más sobresaliente del que se ocupó este estudioso fue la diferencia entre las "milpas de la montaña" (de jamaltic) y las "milpas de abajo" (de alantic). Si bien puede asumirse que todos los suelos de la región estudiada eran aptos para las milpas, en términos generales, se propuso que las milpas de abajo eran más productivas que las de arriba. Así que en las tierras altas de Aguacatenango, Amatenango y Chanal predominaban los cultivos de huertas con plantas herbáceas, tanto ornamentales como medicinales, combinadas con maíz y frijoles. En cambio, varios kilómetros más abajo, los sitios de Venustiano Carranza presentaban un contraste marcado. Aquí, más que jardines, uno se encontraba con huertas que mezclaban árboles frutales, ornamentales y de bebidas — como café- con sembradíos ocasionales de maíz."

En otro ensayo comparativo John Hotchkiss inició por distinguir tres tipos fisiográficos de tierras, que son las de pastoreo, las de temporal y las arables -y a menudo irrigables-. A renglón seguido el autor procedió a identificar las diferentes unidades sociales de tenencia de la tierra. En primer lugar fue ubicada la comunidad total, la que en algunos lugares - como Amatenango y Aguacatenango- estaba representada por las autoridades comunales y en otros - como Teopisca o Venustiano Carranza- por el aparato municipal. En segunda instancia fue colocada la comisión local ejidal, que en comunidades no corporadas tenía un papel crucial en el manejo de asuntos relativos a la tenencia de la tierra. Los barrios y los parajes eran una tercera instancia con funciones de tenencia de la tierra. Una cuarta unidad de gestión de este tipo de asuntos, de acuerdo con Hotchkiss, era el linaje o la familia extensa. En último lugar se encontraba la unidad de mayor importancia, pues era la que realmente trabajaba la tierra en todas las comunidades, es decir, el hogar - o la familia-. ${ }^{2}$

En términos concretos, John Hotchkiss aclaró que en Chanal, Amatenango y Aguacatenango el ayuntamiento era el encargado de supervisar las tierras comunales y emplear los procedimientos legales correspondientes para aumentar los terrenos ejidales. Si bien en Venustiano Carranza y Teopisca, lugares con población indígena y ladina, muchas de las tierras habían caído bajo control privado ladino, aquí también existían algunos terrenos comunales. En Teopisca muchas tierras de pastoreo eran de tipo comunal, mientras que en Carranza lo eran la gran mayoría de terrenos dedicados a los cultivos de temporal. En ambos lugares los ladinos se habían apoderado, por compra y por otros medios, de las tierras mejor 
irrigadas y de extensas áreas para la empresa ganadera. Adicionalmente, aunque la organización de Bienes Comunales, en Carranza, y la Comisión de Ejidos, en Teopisca, eran agencias gubernamentales que debían operar a favor de los ejidatarios - muchos de los cuales eran indígenas-, en gran medida estas instancias favorecían a los ladinos, quienes por cierto controlaban los gobiernos municipales. ${ }^{13}$

\section{Los procesos históricos}

Asumiendo una perspectiva histórica, Robert Adams y Norman McQuown produjeron un documento en el que señalaron varios aspectos que apuntaban a comprender el cambio en las comunidades. En primer lugar, los investigadores resaltaron las migraciones que el área de estudio recibió desde pueblos como Chamula, Huixtán y Oxchuc, las cuales habrían introducido cambios culturales y lingüísticos en un pasado reciente - probablemente a inicios del siglo XX-. El antropólogo y el lingüista identificaron un segundo factor que pudo haber inducido cambios culturales. Por ejemplo, Villa Las Rosas (antes Pinola) se habría transformado de un pequeño pueblo indio y aislado en un centro comercial con población mezclada en la última parte del siglo XIX. Dicha transformación habría sido provocada por la producción a gran escala de caña de azúcar y café en las tierras de las laderas y de los valles bien irrigados abajo del pueblo. ${ }^{14}$

Como parte de este interés general por la historia de las poblaciones y sus transformaciones, Kent Flannery preparó unas sugerencias sobre posibles temas de investigación a desarrollar con relación a la antropología física en la región de estudio. El autor resaltó el trabajo pionero de Frederick Starr sobre los pueblos tseltales y tsotsiles. Este investigador de la Universidad de Chicago presentó sus resultados en 1902 bajo el título The Physical Characters of the Indians of Southern Mexico. Por su parte, el entonces joven y poco conocido Kent Flannery propuso tomar nuevas muestras a las poblaciones y compararlas con las investigaciones anteriores, además de estudiar la dieta, los patrones de asentamiento y las migraciones. ${ }^{15}$
Las sugerencias de Kent Fannery fueron parcialmente cubiertas por la investigación arqueológica. La metodología empleada por Robert Adams en su reconocimiento arqueológico consistió en establecer una secuencia cerámica para Los Altos de Chiapas. Uno de los principales hallazgos de esta investigación fue que, de acuerdo con el desarrollo cultural de Los Altos de Guatemala o del valle cercano del río Grijalva, y con la historia subsecuente de estos dos lugares, Los Altos centrales de Chiapas mostraban una notable escasez de vestigios para los periodos Pre-Clásico y ClásicoTemprano. ${ }^{16}$

En lugar de lo anterior, se propuso que en Los Altos de Chiapas, después del periodo Clásico Temprano - alrededor del año 1000 d.C.-, ocurrió un rápido incremento en la cantidad y densidad de asentamientos humanos. Al parecer, este proceso respondió a la llegada de población que no estaba asentada allí con anterioridad y coincidió aproximadamente con la estimación glotocronológica de Norman McQuown acerca de la separación de las lenguas tseltal y tsotsil. Aquello representaría el advenimiento en el área de los padres lingüísticos a partir de los cuales ese par de lenguas se habrían diferenciado localmente. ${ }^{17}$

El periodo de mayor ocupación del área ocurrió en los niveles temporales Clásico Tardío y PostClásico Temprano, y casi todos los asentamientos estaban caracterizados por ubicarse en promontorios o colinas fácilmente defendibles. Este patrón sugiere que el nivel de organización política dominante para el área y el periodo fueron las comunidades independientes y pequeñas, en lugar de los grandes grupos de asentamientos organizados a nivel regional. Curiosamente, las laderas inferiores de las terrazas de Los Altos fueron poco ocupadas durante el mismo periodo. Las razones ofrecidas para explicar dicho comportamiento fueron principalmente dos: por una parte, la escasez de colinas escarpadas o formaciones de cresta en elevaciones adyacentes a tierras agrícolas bien irrigadas; por otra, la mayor probabilidad de que en las tierras más bajas se presentaran hostilidades con los pueblos no mayas que se movían de arriba a abajo por el valle del Grijalva. ${ }^{18}$ 
Los asentamientos defensivos, que dominaron por un buen periodo de tiempo el área de estudio, ya habían sido abandonados al momento de la Conquista española. En lugar de aquellos, los españoles se encontraron con un pequeño número de agrupaciones políticas más poderosas, las cuales estaban asentadas en mesetas y valles más grandes y estratégicos. Los sitios encontrados cerca de Copanaguastla, Pinola, Chamula, Zinacantán, Comitán y Huixtán son muestras claras de este tipo de asentamiento. ${ }^{19}$

El valle del actual río Grijalva mostró unas características diferentes a las ya descritas para Los Altos y las terrazas cercanas. Tal parece que a lo largo de la zanja del Grijalva el poblamiento fue marcadamente más temprano y denso. Este patrón estuvo asociado con la importancia que tenía el río en tanto que arteria de comunicación. La propuesta es que la subregión baja tuvo un patrón de poblamiento dual. Normalmente una parte de la población residía en aldeas dispersas adyacentes a las principales áreas cultivables en los suelos del valle. Y podría suponerse que los centros nucleados se correspondían en cierto modo con el modelo de "pueblos vacíos", en los que su población permanente era pequeña, aunque eran bastante ocupados en ocasiones ceremoniales o en momentos en que amenazaban las hostilidades. ${ }^{20}$

Edward Calnek presentó una revisión de la bibliografía que hasta entonces se había ocupado de Los Altos de Chiapas antes y después de la Conquista. El autor recalcó que hasta ese momento era escaso el conocimiento que se tenía del período colonial. En lo referente al periodo previo a la Conquista, y acudiendo principalmente a los cronistas Francisco Ximénez, Bernardino de Sahagún, Antonio de Remesal y Bernal Díaz del Castillo, este investigador resaltó el conocimiento relativo a un par de incursiones mexicas en la región de estudio: por una parte, la migración de un grupo náhuatl - los pipiles - entre los siglos IX y X; por otra, el intento de conquista de Comitán por parte de la Triple Alianza durante el reinado de Ahuitzotl $(1486-1502){ }^{21}$

En cuanto a la Conquista propiamente dicha y los primeros años de la Colonia, Edward Calnek pasó revista por temas tales como la actuación de los encomenderos y la disposición de la tierra y del trabajo indio, y por otros como la reducción y desaparición de varios pueblos de indios a causa de las epidemias, lo que de manera concomitante condujo al crecimiento de otras poblaciones. Uno de los aspectos destacados por este investigador es la resistencia que los indios mostraron a los proyectos evangelizadores de los españoles: además del levantamiento indio de 1712, también es mencionada la insurrección chamula de 1869 y el intento fallido de revuelta en $1915 .^{22}$

Muriel Verbitsky y John Hotchkiss redactaron uno de los pocos ensayos, de los producidos en esta primera etapa del proyecto, que explícitamente se dedicó a abordar problemáticas históricas. El punto de partida fue el reconocimiento de que muchas de las características de corporatividad observadas en los pueblos, al momento de realizarse la investigación, eran en buena medida resultado de la política de reducción implementada por el gobierno colonial español. Un segundo aspecto, de no menor importancia, tenía que ver con el paulatino abandono y expulsión de los ladinos de sus propiedades agrícolas ubicadas en las regiones más altas, un fenómeno que pudo haber iniciado a mitad del siglo XIX pero que definitivamente se aceleró en tiempos revolucionarios, en el temprano siglo XX. Se supone que una serie de factores - entre los que se incluían la redistribución de tierras y la construcción de carreteras - habían disminuido el monopolio que los ladinos ejercían sobre el comercio de las tierras altas. Este cambio habría provocado que dicho grupo de población se trasladara a las tierras templadas y cálidas para adelantar sus actividades comerciales. ${ }^{23}$

Además de los factores generales anteriormente señalados, Muriel Verbitsky y John Hotchkiss formularon una clasificación de los pueblos de la región de estudio de acuerdo con el grado y la velocidad de ladinización que habían experimentado hasta ese entonces. Para estos autores resultaba evidente que las "comunidades indígenas" de Aguacatenango, Amatenango, Villa Las Rosas y Venustiano Carranza habían existido desde hacía mucho tiempo y que probablemente las cuatro habían sido establecidas 
durante el periodo de la reducción colonial. Sin embargo, con el paso del tiempo, sólo Amatenango y Aguacatenango habían retenido su autonomía y mantenido su "forma de vida indígena" distintiva a través de los mecanismos de la "comunidad corporada”. En cambio, el largo período de contacto cercano entre indígenas y ladinos, compartiendo el mismo lugar de tierras bajas en Venustiano Carranza, había resultado en el abandono de algunas de las características "indígenas corporadas", de las cuales los autores tenían evidencia que estaban presentes en un primer momento. Y, por último, las tendencias más recientes de poblamiento habían conducido a los ladinos a Villa Las Rosas, y la "corporatividad de esta comunidad indígena” había sido eclipsada casi por completo, lo que resultó en una rápida aculturación de la población indígena en este lugar. ${ }^{24}$

Nodebemos pasarporalto un importante cambioque Muriel y John identificaron en su análisis comparativo: las migraciones. Ellos desconocían desde hacía cuánto tiempo se habían producido tales movimientos e intuían que había existido un flujo constante de población desde las tierras altas hacia la zona de las terrazas en descenso al valle del río Grijalva. No obstante los anteriores vacíos, los investigadores propusieron que cuando los indígenas migraban como familias individuales a trabajar, bien como peones en las fincas o como prestadores de servicios en los pueblos, tendían a ladinizarse - debido al pequeño número de personasEn cambio, cuando los indígenas migraban en grupos grandes y obtenían tierras ejidales para cultivar, tenían mayores posibilidades de conservar sus características sociales y culturales "tradicionales". 25

\section{Las lenguas}

Hubo un trabajo que resumió lo que Yvonne Hajda, Marvin Mayers y Norman McQuown denominaron como la primera descripción estructural que se había hecho hasta entonces de alguno de los dialectos de las lenguas tseltal y tsotsil. La metodología empleada para tratar la información combinó la isoglosa — que permite distinguir características a partir de las cuales se pueden establecer límites entre dialectosy la lexicoestadística, la cual genera un estimativo del momento en el cual dos variantes lingüísticas se desprendieron de un tronco común. ${ }^{26}$

El análisis de los datos también condujo a que los investigadores formularan unos índices de aculturación de acuerdo con los préstamos recibidos del idioma español. Así, en una escala de mayor a menor grado de aculturación, fueron ubicados primero los pueblos de Venustiano Carranza y Villa Las Rosas, pasando luego por los de Amatenango del Valle y Agucatenango, hasta llegar por último al de Chanal. ${ }^{27}$

Para este estudio sobre la distancia dialectal de las lenguas tseltal y tsotsil Norman McQuown combinó varias metodologías. El reconocimiento preliminar hizo uso de las listas de cien y doscientas "palabras básicas" elaboradas por Mauricio Swadesh, acompañadas con una lista de cuatrocientas "palabras culturales" adicionales. A estas seiscientas palabras fueron agregados vocabularios más especializados acerca de la fauna, la flora y la geografía. ${ }^{28}$

Ese reconocimiento preliminar permitió trazar las isoglosas, o líneas imaginarias, entre dos o más áreas geográficas que se distinguían por un rasgo dialectal concreto, ya se tratara de la fonología, el léxico u otro asunto. A partir de esa distinción básica de dialectos en la región tseltal-tsotsil, el equipo de investigación estimó que la distancia mínima entre ellos era de cien años, mientras que la máxima llegaba a 1500. La distancia dialectal más corta encontrada fue entre dos barrios al interior del pueblo de Venustiano Carranza; la más extensa fue trazada entre dos entrevistados, uno del barrio Hamaltik, en Chanal, y el otro del barrio de San Sebastián, en Carranza. ${ }^{29}$

\section{La organización social}

En un artículo comparativo Muriel Verbitsky analizó las diferencias entre las comunidades corporadas y las no corporadas de acuerdo con el grado de ladinización. En Teopisca, por ejemplo, algunos indicadores del proceso de cambio eran de un patrón de residencia patrilocal a uno de tipo neolocal, o el abandono, 
por parte de los indígenas, del trabajo agrícola para dedicarse a oficios comúnmente realizados por los ladinos. Muriel trazó una línea clara de división entre las comunidades corporadas y aquellas que no lo eran. Dentro de la primera categoría englobó a pueblos como Chanal, Amatenango y Aguacatenango, los cuales se caracterizaban por la homogeneidad cultural, la residencia permanente en la cabecera para tener acceso a las tierras y el servicio a la comunidad, entre otros elementos distintivos; mientras que pueblos como Venustiano Carranza y Teopisca entraban en el segundo tipo de comunidad, pues existían amplias diferencias de riqueza entre sus habitantes indígenas y la incidencia de la brujería era mucho menor. ${ }^{30}$

Otro factor que distinguía a ambos tipos de comunidades era la disponibilidad de tierra. Aguacatenango, Chanal y Amatenango tenían más terrenos para cultivos de subsistencia a su disposición, en razón al número de habitantes, que Venustiano Carranza o Teopisca, donde la presencia de ladinos -quienes ejercían control sobre los suelos de mejor calidad - incrementaba la escasez de este recurso. Según Muriel Verbitsky, el bilingüismo era otro indicador del grado de aculturación de una población, pues resultaba ser una expresión del mayor contacto con ladinos, de la presencia de escuelas y de la influencia del Instituto Nacional Indigenista. ${ }^{31}$

Barbara Metzger realizó un trabajo comparativo de los sistemas de parentesco en Aguacatenango, Cancuc y Oxchuc. La investigadora inició por presentar unas generalidades de Chiapas y la región de estudio. En principio ella estableció una clara distinción entre los habitantes de Los Altos y los de la Depresión: mientras en la primera zona la mayoría de ellos eran hablantes de lenguas mesoamericanas y se identificaban como indígenas, en la segunda había una mayoría de población ladina dedicada a la producción agrícola a gran escala, y además acá los pocos indígenas constituían subcomunidades tendientes al bilingüismo y la ladinización. Aguacatenango, Cancuc y Oxchuc están ubicados en Los Altos y en cada uno de ellos se hablaba una variedad de la lengua tseltal. No obstante, el último de los tres pueblos se distinguía por tener una cantidad considerable de población ladina, alrededor de quinientas personas. ${ }^{32}$

Había otros elementos que compartían Aguacatenango, Cancuc y Oxchuc, según las observaciones de Barbara Metzger. Las tres localidades se dedicaban a la agricultura en pequeña escala, lo cual les permitía generar unos pocos excedentes que eran intercambiados con otros pueblos por algunos bienes de primera necesidad. A su vez, la acumulación de riquezas no era bien vista, por lo que era contrarrestada con el requisito de efectuar grandes gastos en el mantenimiento de cargos públicos, y se evitaba para no ser objeto de brujería. Las personas se hacían vulnerables a la brujería por tener demasiado dinero, por insultar a alguien que tenía un nagual o por romper alguna otra regla de buena conducta. De igual modo, las cuevas y las cruces eran centros de importancia religiosa capital en las ceremonias de siembra y cosecha. ${ }^{33}$

Desde luego que Barbara Metzger también identificó puntos de separación entre los tres pueblos. De entrada, cada una de las comunidades tenía un vestido y una identidad propios. Adicionalmente, existía una distancia física y psicológica considerable que dificultaba las relaciones estrechas entre los tres lugares. De acuerdo con las listas de palabras propuestas por Mauricio Swadesh, los datos obtenidos por Marianna Slocum y las estimaciones de Norman McQuown, había 850 años de separación lingüística entre Aguacatenango y Oxchuc. Y si bien este último pueblo era vecino al de Cancuc, al cual alquilaba tierras y le ofrecía sus brazos para trabajar, las dos eran comunidades endogámicas y separadas. ${ }^{34}$

Luego de sopesar varios aspectos, Barbara Metzger concluyó que en Aguacatenango había un grado mayor de aculturación que en Cancucu Oxchuc. Tal deducción se desprendía de elementos tales como el consumo de aguardiente, el uso creciente del vestido ladino o la ausencia de matrimonio infantil en el primero de los pueblos. Y aunque los propios aguacatecos sostenían que los cambios habían llegado con la carretera Panamericana, la antropóloga ofreció varios argumentos que cuestionaban la consideración de los caminos en sí mismos como causantes de la aculturación. Ella 
misma observó que en el pueblo vecino de Amatenango, también cercano a la carretera, eran menores los cambios ocurridos. De igual modo, Barbara notó que tampoco podía decirse que la apertura al mundo exterior por sí sola conducía a la aculturación. Desde hacía algún tiempo había presencia de una población ladina de buen tamaño residente en Oxchuc, como también de trabajo estacional en las fincas de allí y, en menor medida, en las de Cancuc. Después de todo, al momento de analizar los vocabularios culturales, era en Aguacatenango donde existía mayor evidencia de contacto con la cultura española. ${ }^{35}$

Ahora bien, Barbara Metzger comparó las estructuras sociales de los tres pueblos en términos de un supuesto modelo común, particularmente lo que George Murdock denominó como Omaha Normal, es decir, un sistema de parentesco unilateral que tenía términos distintos para el hermano de la mamá y el hermano del papá. Ella fue bastante crítica con respecto a este modelo y de entrada recalcó que era necesario recabar material histórico porque no podía asumirse que Aguacatenango, Cancuc y Oxchuc representaban tres etapas de un mismo proceso de transformación. Al contrario, el punto de partida de esta investigadora fue considerar que, al estar bastante alejados de otros pueblos mayas, era de esperarse que los tseltales y tsotsiles mostraran muchas innovaciones en sus sistemas de parentesco. ${ }^{36}$

La antropóloga identificó varios cambios en cuanto a la estructura social de los tres pueblos. En ese orden de ideas, la antropóloga señaló la pérdida de las reglas de residencia - de patrilocal a neolocal—, la desaparición de funciones de los grupos consanguíneos, la compra y venta de tierras tanto al interior como al exterior de los linajes, así como su posesión por parte de familias y particulares, y una falta de respeto hacia los hombres más viejos y poderosos, con lo que la solución de disputas y la impartición de castigos pasó a ser prerrogativa del gobierno municipal. Más allá de lo cuestionable que puede resultar apegarse a la supuesta existencia de un modelo previo o común de organización social para los tres pueblos, el aporte más importante de este ensayo de Barbara Metzger radica precisamente en mostrar el debilitamiento del principio de linaje, así como también la manera como las terminologías de parentesco cambian, aspectos que además pueden transformarse en diferentes direcciones. ${ }^{37}$

John Baroco escribió tres artículos en los que entrecruzó asuntos de los apellidos, el parentesco y la estructura social. En primer lugar, el investigador redactó unas notas sobre los apellidos en Chanal como parte de un ejercicio de interpretación de algunas fuentes de tipo histórico. El punto de partida fueron las características del pueblo en el momento en que se efectuó la investigación: una mayoría indígena que controlaba el gobierno local y con un notable monolingüismo en tseltal. Como muchas otras comunidades indígenas de Chiapas, Chanal se caracterizaba por tener dos barrios endogámicos y, al interior de cada uno de ellos, un conjunto de clanes exógamos. Según este antropólogo, un apellido indígena no se combinaba más que con un apellido español. Así, una persona cuyo supuesto nombre fuera Antonio Hernández Yemoc, sería miembro del clan Hernández y del linaje Yemoc o de la subdivisión de aquel clan. De acuerdo con la costumbre, a esta persona se le prohibía casarse con una mujer de apellido Hernández. ${ }^{38}$

El objetivo del primer informe presentado por Baroco fue mostrar una manera de acercarse a esos grupos de parentesco, así como evidenciar los problemas que podían suscitarse. Las fuentes empleadas para el estudio provenían de la Oficina del Registro Civil de San Cristóbal de Las Casas y se trataba fundamentalmente de las actas de matrimonios, nacimientos y defunciones. Uno de los principales hallazgos de John Baroco fue identificar un cambio en la forma de registro: los dos apellidos indígenas del padre fueron reemplazados por el apellido español del padre seguido del de la madre. Para sortear dicha dificultad, el autor propuso atender al hecho de que Chanal era una rama que se había desprendido de la comunidad de Oxchuc hacía menos de doscientos años y, por tanto, había que seguir las pistas a través de los nombres en esa última localidad. ${ }^{39}$

El segundo reporte de John Baroco se ocupó del uso de los nombres calendáricos mayas en algunos pueblos de Chiapas a través de testimonios del siglo XVI. Este 
trabajo es particularmente interesante -y el más conocido del autor- por emplear registros bautismales, matrimoniales y de cofradías correspondientes a los años comprendidos entre 1557 y 1584. Entre las peculiaridades que presenta esta fuente antigua se encuentra haber iniciado el registro de información en el pueblo de Copanaguastla —-desaparecido en el siglo XVII-y finalizar en el de Comitán. ${ }^{40}$

Seguramente, una de las principales razones por las que este trabajo de John Baroco fue empleado por varios investigadores que lo sucedieron es la interesante discusión que él presentó acerca de la lengua o las lenguas en que están escritos tres pasajes del documento en cuestión: bien fueran coxoh, tseltal, tsotsil o tojolabal. Entre las localidades mencionadas a lo largo de las fojas se encuentran algunas ya desaparecidas, como Coapa y Pantla, correspondiendo la primera de ellas a un pueblo que se ubicaba al otro lado de Comitán, y la segunda a una parcialidad que fue incorporada al pueblo de Socoltenango. ${ }^{41}$

John Baroco concluyó que en los primeros años que transcurrieron tras la Conquista española los nombres calendáricos — varios de ellos tseltales y yucatecasaún se encontraban en pleno uso en los registros parroquiales de los alrededores de los pueblos de Comitán y Copanaguastla. Finalmente, este investigador se inclinó por considerar que la lengua en que estaban escritos los pasajes del libro era tsotsil y que había sido iniciado en el convento de Copanaguastla. ${ }^{42}$ Si bien puede ser posible que este último lugar haya sido el origen del documento, cabe señalar que, a la luz de trabajos históricos cercanos a nuestros días, la lengua principal hablada en Copanaguastla era el tseltal (Ruz, 1985).

Además de los dos trabajos anteriormente reseñados, John Baroco preparó un tercer escrito para el reporte presentado por el proyecto Chicago en 1959. Este documento — hasta donde se sabe - jamás fue publicado y se ocupó de describir el sistema de apellidos compuestos y relacionarlo con los cambios en la estructura social. Después de todo, el último objetivo de esta tripleta de ensayos fue sentar las bases para una investigación histórica sólida que pudiera determinar la existencia o no de clanes en el área de estudio y sus posibles transformaciones a lo largo del tiempo. John Baroco también notó que en algunos pueblos existían grupos exógamos - a la manera de linajes-, mientras que en otros no. Además, encontró que la explicación más usual para esa diferencia se centraba en los supuestos grados de aculturación. La intención en este ensayo fue presentar cuatro casos diferentes de ausencia y presencia de grupos exógamos, analizados a partir de la relación entre la estructura social y el uso de apellidos. ${ }^{43}$

El primer caso estudiado es el de San Bartolomé de Los Llanos, donde John Baroco identificó que los barrios cumplían un papel destacado en la organización política, religiosa y agrícola. Sin embargo, un aspecto que había cambiado en ese pueblo era que el nagualismo había entrado en claro desuso, comportamiento que estaba relacionado con la desaparición de líderes representativos. Otro elemento muy característico del San Bartolomé de aquellos años era el sistema de nombres que combinaba un apellido español con uno indígena. Si bien el apellido indígena servía para indicar la procedencia de la persona a través del linaje, este último ya no cumplía ninguna función política o religiosa en la comunidad..$^{44}$

A continuación, John Baroco pasó a identificar las particularidades que mostraba el pueblo de San Pablo Chalchihuitán. A diferencia de San Bartolomé, el pueblo de San Pablo se caracterizaba por tener una cabecera vacía. Los dos lugares compartían la pérdida del peso que los naguales habían tenido en el pasado en asuntos relativos al control social. En Chalchihuitán también existían los nombres compuestos. Esta última peculiaridad hizo pensar al antropólogo que San Bartolomé tenía un grado de aculturación mayor que San Pablo, pues en este último pueblo el apellido indígena estaba ligado no sólo a linajes sino también a barrios, o calpules, particulares. ${ }^{45}$

El tercer caso que llamó la atención de John Baroco fue el de Oxchuc. Uno de los rasgos más sobresalientes de esta localidad era la importancia organizativa que tenían sus dos barrios. Estas unidades no sólo regían las poblaciones y tierras, sino que también controlaban las actividades políticas y religiosas por medio de una 
jerarquía de cargos. A diferencia de San Bartolomé y San Pablo, en Oxchuc quienes ejercían dichos oficios eran poseedores de poderosos naguales que contribuían a mantener el orden y castigar a los transgresores. En este pueblo, como también en los dos anteriores, los nombres compuestos también estaban presentes. No obstante, en el caso de Oxchuc los apellidos estaban ligados a linajes, clanes y calpules, donde los líderes de cada unidad ejercían su autoridad y control social a través de la fuerza de sus naguales. ${ }^{46}$

El último pueblo considerado en este ejercicio comparativo fue San Juan Cancuc. John Baroco afirmó que en esa localidad el apellido indígena estaba asociado a un barrio, si bien esta unidad organizativa no tenía límites espaciales precisos ni tampoco era endógama. $\mathrm{Y}$, aunque estaba presente la creencia en espíritus guardianes, parecía no existir ya una jerarquía de cargos con líderes que usaran el poder de los naguales para impartir justicia. ${ }^{47}$

Bajo el supuesto de que en algún momento existió un patrón común de organización social caracterizado por barrios, o calpules, endógamos y clanes exógamos, el autor concluyó que en las cuatro comunidades señaladas el apellido indígena hacía referencia al linaje. En cambio, ya no había claridad de que el apellido en español hiciera referencia a los clanes. Teniendo en cuenta este último punto, y ante la falta notable de investigaciones históricas, John Baroco planteó algunas hipótesis de trabajo. No descartó que el "calpul azteca" hubiera sido una forma de organización impuesta por parte del gobierno colonial español o de los propios grupos indígenas del Valle de México. Otra posibilidad era que los barrios fueran el resultado de la política de reducciones implementada por la Corona española para concentrar la población indígena desperdigada en los campos y que cada calpul correspondiera a una comunidad anteriormente separada. ${ }^{48}$

Una sugerencia final que planteó John Baroco tenía que ver con el origen de los apellidos españoles que llevaban los indígenas. El antropólogo recordó que una práctica común en los primeros años de la conquista española consistió en oficiar bautizos colectivos en los cuales un grupo de personas —independientemente de su procedencia - recibía el mismo nombre del patrocinador de la ceremonia. Lo anterior implicaba, o bien que el nombre español dado al conjunto de personas pudo haber coincidido con un clan o calpul, o bien que en el acto se creara un parentesco artificial entre los bautizados, con la consecuente prohibición de matrimonio entre ellos. ${ }^{49}$

\section{Algunas reflexiones finales}

No es que fuera inexistente la antropología relativa a Chiapas antes de la década de 1960. Basta con recordar las excelsas obras de Calixta Guiteras y Ricardo Pozas como muestras de investigaciones basadas en trabajo de campo intensivo. Sin embargo, hasta mediados del siglo $\mathrm{XX}$ eran pocos los estudios que se habían realizado en el área, destacando la escasez de trabajos lingüísticos, como también de monografías dedicadas a los pueblos. En este sentido, uno de los principales aportes del proyecto emprendido por la Universidad de Chicago entre 1956 y 1959 fue iniciar un estudio que abarcó gran amplitud geográfica, atendió las variaciones regionales, incorporó datos cuantitativos y combinó diferentes disciplinas (Medina, 2015).

A primera vista podría decirse que los investigadores del proyecto Chicago ofrecieron una visión muy esquemática de las poblaciones de la región tseltaltsotsil, basada en los grados de asimilación cultural, que ubicaba a unas comunidades como corporadas, a otras como abiertas y a las restantes en un lugar intermedio. No obstante, el propio desarrollo de sus investigaciones los condujo a reformular muchas de sus suposiciones iniciales.

Si bien la originalidad del proyecto consistió en ser el primer estudio dialectal de la variación del habla en grupos indígenas, a la postre se convirtió en una empresa que reunió las habilidades de investigadores formados en diferentes disciplinas, cualidad que permitió atender numerosas variables y dimensiones de la realidad humana local. De este modo, fueron claramente descritas las múltiples formas de habitación y actividad productiva, dependiendo de la altitud en que se encontraban ubicados los terrenos. Aspectos 
como los tipos de tierra, las unidades de tenencia y la transferencia de las mismas también aparecieron en varios de los análisis. Entre los resultados más destacados de la empresa académica puede aludirse a la identificación de factores clave para la explicación de los cambios ocurridos, entre ellos: las migraciones, el cultivo de la caña y del café o la interacción entre indígenas y ladinos.

Hubo varios aportes para la comprensión de los procesos históricos. Los hallazgos en el conocimiento del periodo prehispánico fueron sorprendentes. Había claras diferencias entre Los Altos, las Terrazas y los Valles Centrales de Chiapas. En Los Altos el tipo de poblamiento fue tardío (aproximadamente 1000 d.C.) y consistió en pequeños asentamientos defensivos ubicados en promontorios. Por su parte, las Terrazas fueron poco ocupadas debido a la escasez de colinas y la gran exposición a ataques enemigos. En cambio, los Valles Centrales tuvieron una ocupación temprana y relativamente densa, consistente en un patrón dual -o de "pueblo vacío" - que combinaba las aldeas dispersas con centros nucleares.

Pese a que no fue claramente documentado, una de las fortalezas del proyecto es haber propuesto algunas problemáticas históricas atendiendo a una perspectiva de larga duración. En esta línea de investigación cobraban importancia elementos tales como la política colonial de reducciones, un supuesto desplazamiento de los ladinos desde las tierras altas hacia la Depresión Central a mediados del siglo XIX y las migraciones en general entre ambos puntos de la geografía chiapaneca.

Otro importante hallazgo de las investigaciones fue revelar algunos de los diferentes cruces producidos entre lengua e identidad. Una de las principales novedades de la empresa académica fue elaborar la primera descripción de los dialectos de las lenguas tseltal y tsotsil combinando listas de palabras con la isoglosa y la léxico-estadística. Esta misma metodología permitió construir índices de aculturación partiendo de los préstamos lingüísticos.

Uno de los aportes de los trabajos fue mostrar la diferencia entre pueblos con barrios duales y múltiples, así como la importancia de estos conglomerados en la organización social. Precisamente uno de los resultados consistió en haber estudiado los grados de ladinización atendiendo a patrones de residencia, disponibilidad de tierras, formas de trabajo y presencia de bilingüismo.

Muchos pueblos tseltales, por ejemplo, compartían aspectos como la agricultura de subsistencia, la poca acumulación de riqueza, la preponderancia de la brujería y los naguales y la práctica de rituales en cuevas y lugares con cruces. Sin embargo, existían claras diferencias en términos del vestido y la identidad, como también de la distancia geográfica, cultural y lingüística. En lugar de un enfoque apegado a diferentes etapas evolutivas, ellas y ellos recalcaron la necesidad de emprender estudios históricos que tuvieran como punto de partida la consideración de que los pueblos tseltales y tsotsiles tenían sistemas de parentesco con características particulares.

Hubo quienes atendieron las transformaciones sociales que estaban ocurriendo a lo largo del tiempo. Un objeto de análisis fue la combinación entre apellidos españoles e indígenas y su relación con la estructura social de las poblaciones. Otro aporte significativo fue identificar las diferentes combinaciones que se daban entre variables tales como el tipo de asentamiento, la estructura de los barrios, las formas de tenencia de la tierra, las unidades de parentesco, la creencia en los naguales, el ejercicio de autoridad, el papel de los ancianos y el tipo de apellidos.

El proyecto señaló la necesidad de cubrir algunos vacíos de conocimiento en el futuro. Recordemos que uno de los aspectos que se resaltaron en varios de los documentos fue la necesidad de emprender estudios históricos.

Uno de los principales reparos que puede hacerse a varios de los trabajos incluidos en este primer reporte es que, si bien la mayoría de los estudiosos reconocían los procesos de transformación que estaban ocurriendo, el punto de partida era un molde purista y poco dinámico para comprender la especificidad de los pueblos de la región. En este sentido, cabría hacer una revisión de la manera en que fueron interpretados los procesos de 
cambio, así como también los factores ofrecidos para darles explicación.

\section{Notas}

${ }^{1}$ Las fuentes de primera mano de este artículo provienen de dos archivos: Microfilm Collection of Manuscripts on Cultural Anthropology, Joseph Regenstein Library, University of Chicago (se utilizarán las siglas MCA cuando se haga referencia a este archivo), y los Sol Tax Papers, Regenstein Library, Special Collections, University of Chicago (se utilizarán las siglas RLSC-ST).

${ }^{2}$ McQuown, N. A. "Overview and Preview", Report on the "Man in Nature" Project (1956-1959), Pt. I, MCA, Series XIV, No. 92.

${ }^{3}$ Report on the "Man in Nature" Project (1956-1959), Department of Anthropology of University of Chicago in the Tzeltal-Tzotzil Speaking Region of the State of Chiapas, Mexico, MCA, Series XIV, Pt. I: No. 92; Pt. II: No. 93; Pt III: No. 94.

${ }^{4}$ Nash, M., Verbitsky, M., Hotchkiss, J. y McQuown, N. "Present-Day Socio-Cultural-Linguistic-Ethnohistorical Correlations", Report on the "Man in Nature" Project (19561959), Pt. I, MCA, Series XIV, No. 92, p. 2.

${ }^{5}$ Nash, M., Verbitsky, M., Hotchkiss, J. y McQuown, N. "Present-Day Socio-Cultural-Linguistic-Ethnohistorical Correlations", Report on the "Man in Nature" Project (19561959), Pt. I, MCA, Series XIV, No. 92, pp. 7-8.

${ }^{6}$ McQuown, N. A. "Overview and Preview", Report on the "Man in Nature" Project (1956-1959), Pt. I, MCA, Series XIV, No. 92, p. 3.

${ }^{7}$ McQuown, N. A. "Overview and Preview", Report on the "Man in Nature" Project (1956-1959), Pt. I, MCA, Series XIV, No. 92, p. 9.

8 Hotchkiss, J. "Chanal, Teopisca and Venustiano Carranza", Report on the "Man in Nature" Project (1956-1959), Pt. II, MCA, Series XIV, No. 93, pp. 1-9.

9 Wagner, Ph. y Hotchkiss, J. "Habitat and Human Activity", Report on the "Man in Nature" Project (1956-1959), Pt. I, MCA, Series XIV, No. 92.

10 Wagner, Ph. "Habitat", Report on the "Man in Nature" Project (1956-1959), Pt. II, MCA, Series XIV, No. 93, pp. 5 y 11.
${ }^{11}$ Kaplan, L. "Vegetation Dynamics", Report on the "Man in Nature" Project (1956-1959), Pt. II, MCA, Series XIV, No. 93, pp. 2-12.

${ }^{12}$ Hotchkiss, J. "Appendix II: Comparative Land Tenure Systems", Report on the "Man in Nature" Project (1956-1959), Pt. II, MCA, Series XIV, No. 93, pp. 1-2.

${ }^{13}$ Hotchkiss, J. "Appendix II: Comparative Land Tenure Systems", Report on the "Man in Nature" Project (1956-1959), Pt. II, MCA, Series XIV, No. 93, pp. 3-5.

${ }^{14}$ Adams, R. y McQuown, N. A. "Pre-History and PostConquest Developments", Report on the "Man in Nature" Project (1956-1959), Pt. I, MCA, Series XIV, No. 92, pp. 6-7.

${ }^{15}$ Flannery, K. "Notes on the Tzeltal-Tzotzil Physical Anthropology", Report on the "Man in Nature" Project (19561959), Pt. II, MCA, Series XIV, No. 93, pp. 1-3.

${ }^{16}$ Adams, R. "Archeological Reconnaissance", Report on the "Man in Nature" Project (1956-1959), Pt. II, MCA, Series XIV, No. 93, p. 9.

17 Adams, R. "Archeological Reconnaissance", Report on the "Man in Nature" Project (1956-1959), Pt. II, MCA, Series XIV, No. 93, p. 10.

${ }^{18}$ Adams, R. "Archeological Reconnaissance", Report on the "Man in Nature" Project (1956-1959), Pt. II, MCA, Series XIV, No. 93, p. 11-14.

19 Adams, R. "Archeological Reconnaissance", Report on the "Man in Nature" Project (1956-1959), Pt. II, MCA, Series XIV, No. 93, p. 16.

${ }^{20}$ Adams, R. "Archeological Reconnaissance", Report on the "Man in Nature" Project (1956-1959), Pt. II, MCA, Series XIV, No. 93, pp. 20-21.

${ }^{21}$ Calnek, E. E. "Ethnohistorical Notes", Report on the "Man in Nature" Project (1956-1959), Pt. II, MCA, Series XIV, No. 93, pp. 1-4.

${ }^{22}$ Calnek, E. E. "Ethnohistorical Notes", Report on the "Man in Nature" Project (1956-1959), Pt. II, MCA, Series XIV, No. 93, pp. 5-9.

${ }^{23}$ Verbitsky, M. y Hotchkiss, J. "Appendix I: Historical Problems", Report on the "Man in Nature" Project (1956-1959), Pt. II, MCA, Series XIV, No. 93, pp. 4-6.

${ }^{24}$ Verbitsky, M. y Hotchkiss, J. "Appendix I: Historical Problems", Report on the "Man in Nature" Project (1956-1959), Pt. II, MCA, Series XIV, No. 93, p. 7. 
${ }^{25}$ Verbitsky, M. y Hotchkiss, J. “Appendix I: Historical Problems", Report on the "Man in Nature" Project (19561959), Pt. II, MCA, Series XIV, No. 93, pp. 7-10.

${ }^{26}$ Mayers, Marvin K., Hajda, Y. y McQuown, N. “TzeltalTzotzil Linguistics: Description, Distributions, Relations", Report on the "Man in Nature" Project (19561959), Pt. II, MCA, Series XIV, No. 93.

${ }^{27}$ Mayers, Marvin K., Hajda, Y. y McQuown, N. “TzeltalTzotzil Linguistics: Description, Distributions, Relations", Report on the "Man in Nature" Project (19561959), Pt. II, MCA, Series XIV, No. 93, p. 7.

${ }^{28}$ McQuown, N. A. "Measures of Dialect Distance in Tzeltal-Tzotzil", Report on the "Man in Nature" Project (1956-1959), Pt. III, MCA, Series XIV, No. 94, p. 1.

${ }^{29}$ McQuown, N. A. "Measures of Dialect Distance in Tzeltal-Tzotzil", Report on the "Man in Nature" Project (1956-1959), Pt. III, MCA, Series XIV, No. 94, p. 4.

30 Verbitsky, M. "Comparative Social Organization of the Transect Communities", Report on the "Man in Nature" Project (1956-1959), Pt. II, MCA, Series XIV, No. 93, pp. 5-6 y 14-16.

31 Verbitsky, M. "Comparative Social Organization of the Transect Communities", Report on the "Man in Nature" Project (1956-1959), Pt. II, MCA, Series XIV, No. 93, pp. 17-18.

${ }^{32}$ Metzger, B. "The Social Structure of Three Tzeltal Communities: Omaha Systems in Change", Report on the "Man in Nature" Project (1956-1959), Pt. III, MCA, Series XIV, No. 94, pp. 1-2.

${ }^{33}$ Metzger, B. "The Social Structure of Three Tzeltal Communities: Omaha Systems in Change", Report on the "Man in Nature" Project (1956-1959), Pt. III, MCA, Series XIV, No. 94, pp. 3-4.

${ }^{34}$ Metzger, B. "The Social Structure of Three Tzeltal Communities: Omaha Systems in Change”, Report on the "Man in Nature" Project (1956-1959), Pt. III, MCA, Series XIV, No. 94, p. 4.

${ }^{35}$ Metzger, B. "The Social Structure of Three Tzeltal Communities: Omaha Systems in Change", Report on the "Man in Nature" Project (1956-1959), Pt. III, MCA, Series XIV, No. 94, pp. 5-6 y 31.

${ }^{36}$ Metzger, B. "The Social Structure of Three Tzeltal Communities: Omaha Systems in Change”, Report on the "Man in Nature" Project (1956-1959), Pt. III, MCA, Series XIV, No. 94, pp. 6-9.

${ }^{37}$ Metzger, B. "The Social Structure of Three Tzeltal Communities: Omaha Systems in Change", Report on the "Man in Nature" Project (1956-1959), Pt. III, MCA, Series XIV, No. 94, pp. 12-30.

38 Baroco,J. V. "El Método Histórico en Antropología Social: Los Apellidos de Chanal", Reporton the "Man in Nature" Project (1956-1959), Pt. III, MCA, Series XIV, No. 94, p. 1.

39 Baroco, J. V. "El Método Histórico en Antropología Social: Los Apellidos de Chanal", Report on the "Man in Nature" Project (1956-1959), Pt. III, MCA, Series XIV, No. 94, pp. 2-6.

${ }^{40}$ Baroco, J. V. "Notes on the use of Calendarical Names in Sixteenth Century Chiapas", Report on the "Man in Nature" Project (1956-1959), Pt. III, MCA, Series XIV, No. 94, pp. 1-5.

${ }^{41}$ Baroco, J. V. "Notes on the use of Calendarical Names in Sixteenth Century Chiapas", Report on the "Man in Nature" Project (1956-1959), Pt. III, MCA, Series XIV, No. 94, pp. 6-8. Pantla y Coapa fueron pueblos de indios de la "primera generación", es decir fundados a raíz de las congregaciones que hicieron los dominicos. Pantla se ubicaba entre las actuales colonias tojolabales de Veracruz y La Libertad, en donde se encuentra todavía un sitio arqueológico. Coapa estaba a unos dieciséis kilómetros al sur de donde ahora se encuentra Tzimol. A raíz de los ataques de los lacandones, los habitantes de Pantla fueron reubicados en Socoltenango, en donde dieron lugar a las parcialidades de Pantla La Grande y Pantilla. Coapa pasó de ser uno de los pueblos más grandes de la provincia a entrar en decadencia. A pesar de varios intentos de refundación, desapareció por completo en la década de 1720 (Viqueira, 1997: Cap. 8, 2ª parte).

${ }^{42}$ Baroco, J. V. "Notes on the use of Calendarical Names in Sixteenth Century Chiapas", Report on the "Man in Nature" Project (1956-1959), Pt. III, MCA, Series XIV, No. 94, p. 9.

${ }^{43}$ Baroco, J. V. "Tzotzil-Tzeltal Nomenclature and Social Structure", Report on the "Man in Nature" Project (1956-1959), Pt. III, MCA, Series XIV, No. 94, pp. 3-5.

${ }^{44}$ Baroco, J. V. "Tzotzil-Tzeltal Nomenclature and Social Structure", Report on the "Man in Nature" Project (1956-1959), Pt. III, MCA, Series XIV, No. 94, pp. 6-7. 
${ }^{45}$ Baroco, J. V. "Tzotzil-Tzeltal Nomenclature and Social Structure", Report on the "Man in Nature" Project (1956-1959), Pt. III, MCA, Series XIV, No. 94, pp. 7-8.

${ }^{46}$ Baroco, J. V. "Tzotzil-Tzeltal Nomenclature and Social Structure", Report on the "Man in Nature" Project (1956-1959), Pt. III, MCA, Series XIV, No. 94, pp. 8-9.

${ }^{47}$ Baroco, J. V. "Tzotzil-Tzeltal Nomenclature and Social Structure", Report on the "Man in Nature" Project (1956-1959), Pt. III, MCA, Series XIV, No. 94, p. 10.

${ }^{48}$ Baroco, J. V. "Tzotzil-Tzeltal Nomenclature and Social Structure", Report on the "Man in Nature" Project (1956-1959), Pt. III, MCA, Series XIV, No. 94, pp. 10-14.

${ }^{49}$ Baroco, J. V. "Tzotzil-Tzeltal Nomenclature and Social Structure", Report on the "Man in Nature" Project (1956-1959), Pt. III, MCA, Series XIV, No. 94, p. 15.

\section{Referencias}

Barrera, Óscar Javier (2016). “San Bartolo y Cuxtepeques: Lengua, tierra y población en la Depresión Central de Chiapas". En Trace, 69: 9-33.

Fábregas, Andrés (2007). "Esther Hermitte y la antropología en Chiapas". En Andrés Fábregas y Rosana Guber (coords.), Chiapas en las notas de campo de Esther Hermitte. San Cristóbal de Las Casas: Universidad Intercultural de Chiapas, Instituto de Desarrollo Económico y Social-Centro de Antropología Social de Argentina, pp. 21-29.

Guber, Rosana (2007). "Dos tesis para Pinola”. En Andrés Fábregas y Rosana Guber (coords.), Chiapas en las notas de campo de Esther Hermitte. San Cristóbal de Las Casas: Universidad Intercultural de Chiapas, Instituto de Desarrollo Económico y Social-Centro de Antropología Social de Argentina, pp. 31-34.

McQuown, Norman A. y Julian Pitt-Rivers (1970). "Prefacio: cambios sociales, culturales y lingüísticos en la sierra”. En Norman A. McQuown y Juian Pitt-Rivers (comps.), Ensayos de antropología en la zona central de Chiapas. México: Instituto Nacional Indigenista, Secretaría de Educación Pública, pp. 9-20.
Medina, Andrés (1983). "Diez años decisivos”. En Andrés Medina y Carlos García Mora (eds.), La quiebra política de la antropología social en México (Antología de una polémica), vol. I: 'La impugnación'. México: Universidad Nacional Autónoma de México, pp. 27-74.

Medina, Andrés (1995). "Presentación (atisbos, cabos sueltos y otras manías)". En Marcelo Díaz de Salas, San Bartolomé de los Llanos en la escritura de un etnógrafo: 1960-1961. Diario de campo. Venustiano Carranza, Chiapas. Tuxtla Gutiérrez: Gobierno del Estado de Chiapas, Universidad de Ciencias y Artes del Estado de Chiapas, pp. 7-49.

Medina, Andrés (2015). “Antropología y geopolítica. La Universidad de Chicago en los Altos de Chiapas: el proyecto Man-in-Nature (1956-1962)". En Andrés Medina Hernández y Mechthild Rutsch (coords.), Senderos de la antropología: discusiones mesoamericanistas y reflexiones históricas. México: Instituto Nacional de Antropología e Historia, Universidad Nacional Autónoma de México-Instituto de Investigaciones Antropológicas, pp. 205-274.

Molina, Virginia (1976). San Bartolomé de Los Llanos. Una urbanización frenada. México: Centro de Investigaciones Superiores en Antropología Social, Instituto Nacional de Antropología e Historia.

Morales Avendaño, Juan María (1986). San Bartolomé de los Llanos en la historia de Chiapas. Tuxtla Gutiérrez: Universidad Autónoma de Chiapas.

Ruz, Mario Humberto (1985). Copanaguastla en un espejo: un pueblo tzeltal en el Virreinato. San Cristóbal de Las Casas: Centro de Estudios Indígenas-Universidad Autónoma de Chiapas.

Viqueira, Juan Pedro (1997). Cronotopología de una región rebelde. La construcción histórica de los espacios sociales en la alcaldía mayor de Chiapas (1520-1720). Tesis del doctorado en ciencias sociales con especialidad en historia y civilizaciones. París: Escuela de Altos Estudios en Ciencias Sociales.

Vogt, Evon Zartman (1994). Fieldwork among the Maya: Reflections on the Harvard Chiapas Project. Albuquerque: University of New Mexico Press. 


\section{Mapa 1. Distrubución y localización de los pueblos tseltales y tsotsiles hacia 1800}

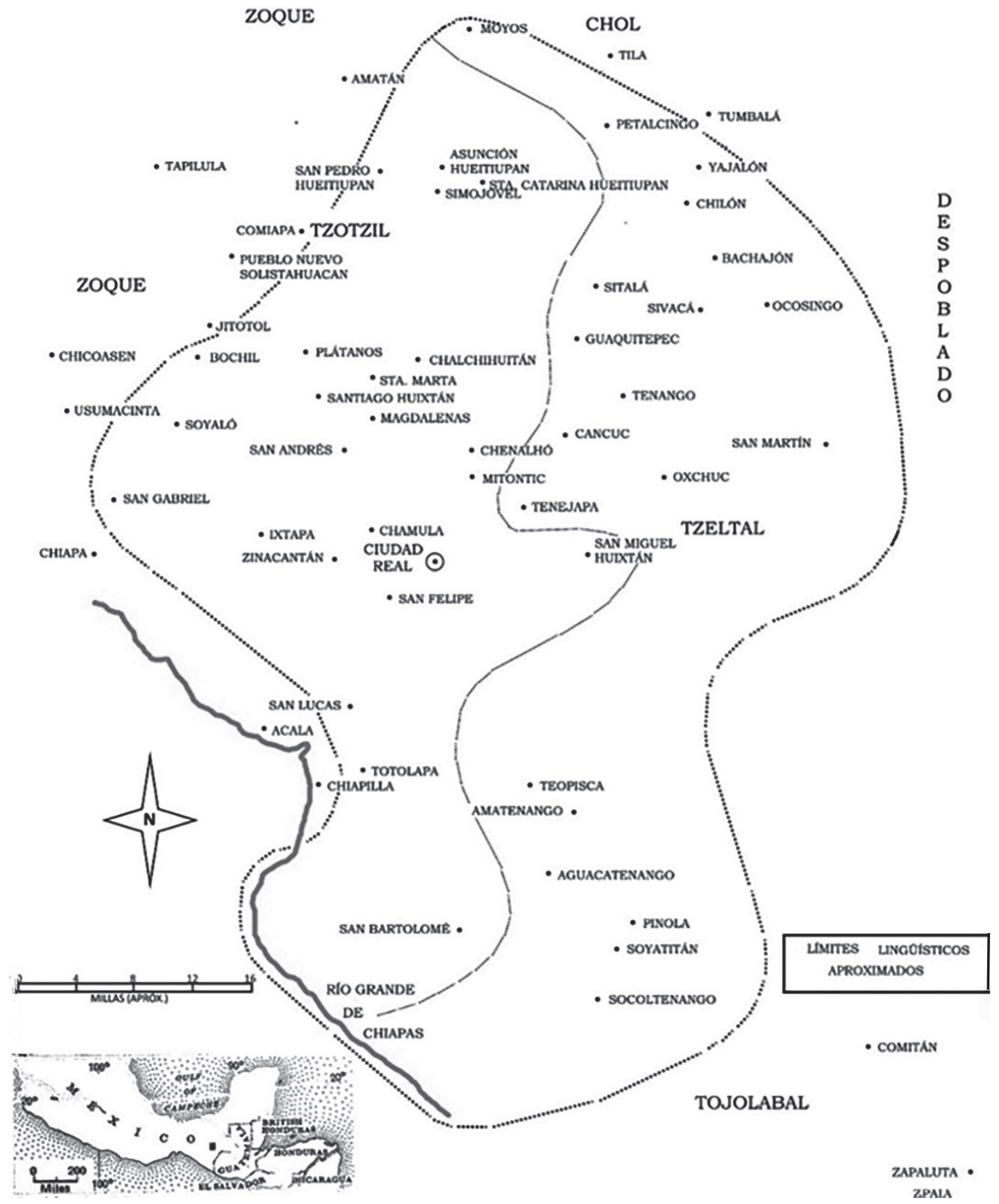

Fuente: Regenstein Library, Special Collections, Sol Tax Papers, University of Chicago, Chicago, caja 104, exp. 5, Edward Calnek, "Distribution and Localization of the Tzeltal and Tzotzil Pueblos of the Highlands of Chiapas from Earliest Times to the Present", 20 de febrero de 1961. 